\title{
Vacuum polarization of graphene with a supercritical Coulomb impurity: Low-energy universality and discrete scale invariance
}

\author{
Yusuke Nishida \\ Department of Physics, Tokyo Institute of Technology, Ookayama, Meguro, Tokyo 152-8551, Japan
}

(Dated: May 2014)

\begin{abstract}
We study massless Dirac fermions in a supercritical Coulomb potential with the emphasis on that its low-energy physics is universal and parametrized by a single quantity per supercritical angular momentum channel. This low-energy parameter with the dimension of length is defined only up to multiplicative factors and thus each supercritical channel exhibits the discrete scale invariance. In particular, we show that the induced vacuum polarization has a power-law tail whose coefficient is a sum of log-periodic functions with respect to the distance from the potential center. This coefficient can also be expressed in terms of the energy and width of so-called atomic collapse resonances. Our universal predictions on the vacuum polarization and its relationship to atomic collapse resonances shed new light on the longstanding fundamental problem of quantum electrodynamics and can in principle be tested by graphene experiments with charged impurities.
\end{abstract}

PACS numbers: 73.22.Pr, 81.05.ue, 03.65.Pm

\section{INTRODUCTION}

Fate of vacuum in a strong Coulomb potential produced by a heavy atomic nucleus is a fundamental problem of quantum electrodynamics and has been the subject of a long study [1]. One of the physical quantities that have attracted particular interest is the induced vacuum polarization [2-6]. It is well known that there are two distinct regimes, subcritical $Z<\alpha^{-1}$ and supercritical $Z>\alpha^{-1}$, depending on the nuclear charge $Z$ relative to the reciprocal of the fine structure constant $\alpha=e^{2} /\left(4 \pi \varepsilon_{0} \hbar c\right) \approx 1 / 137[7]$. Although the resulting physics can be qualitatively different in the two regimes [6], intriguing phenomena caused by the supercritical Coulomb potential remain elusive because of the absence of such superheavy atomic nuclei.

The situation has changed since 2004 when a graphene was successfully isolated [8, 9], which realizes massless Dirac fermions in two dimensions with the Fermi velocity $v_{\mathrm{F}} \approx 10^{6} \mathrm{~m} / \mathrm{s}[10$. Because the corresponding "fine structure constant" is as large as $e^{2} /\left(4 \pi \varepsilon_{0} \hbar v_{\mathrm{F}}\right) \approx 2$, a cluster of charged impurities placed on graphene can produce the supercritical Coulomb potential and thus the resulting intriguing phenomena are now within experimental reach 11 13]. Theoretically, the vacuum polarization of graphene has been studied intensively in both subcritical and supercritical regimes [14 23]. However, in our opinion, the vacuum polarization induced by the supercritical Coulomb potential has not been understood completely even without electron-electron interaction. The objective of this paper is to shed new light on this longstanding fundamental problem.

Noninteracting massless Dirac fermions in two dimensions subject to a Coulomb potential are described by the Dirac equation:

$$
\left(-i \partial \cdot \boldsymbol{\sigma}-\frac{g}{r}\right) \Psi_{\epsilon j}(\boldsymbol{r})=\epsilon \Psi_{\epsilon j}(\boldsymbol{r}) .
$$

Here $\boldsymbol{\partial} \cdot \boldsymbol{\sigma} \equiv \partial_{x} \sigma_{x}+\partial_{y} \sigma_{y}$ is the kinetic term, $\epsilon \equiv E / \hbar v_{\mathrm{F}}$ is the normalized energy, and $g \equiv Z e^{2} /\left(4 \pi \varepsilon_{0} \hbar v_{\mathrm{F}}\right)$ is the dimensionless coupling constant with $-e$ and $Z e$ being the electron and impurity charges, respectively. Because the Coulomb potential is circularly symmetric, the wave function $\Psi_{\epsilon j}(\boldsymbol{r})$ can be chosen as an eigenfunction of the conserved total angular momentum; $j=$ $\pm 1 / 2, \pm 3 / 2, \ldots$ [15, 16]. Accordingly, the vacuum polarization electron density is formally expressed as

$$
n(\boldsymbol{r})=\frac{1}{2} \sum_{\epsilon<0} \sum_{j}\left|\Psi_{\epsilon j}(\boldsymbol{r})\right|^{2}-\frac{1}{2} \sum_{\epsilon>0} \sum_{j}\left|\Psi_{\epsilon j}(\boldsymbol{r})\right|^{2}
$$

assuming appropriate normalization and regularization [24]. While an explicit calculation will be performed below, the functional form of $n(\boldsymbol{r})$ can be deduced only from symmetry and dimensional analysis. In particular, because of the absence of intrinsic scale in the subcritical regime $|g|<1 / 2$, the induced electron density has to be in a scale invariant form and the only possibility is

$$
n(\boldsymbol{r})=N_{0} \delta(\boldsymbol{r})
$$

We note that another apparently possible form $\sim 1 / r^{2}$ is not compatible with the scale invariance because its Fourier transform generates a logarithm which requires some scale [17]. Therefore, the vacuum polarization induced by the subcritical Coulomb potential is localized at the potential center [15 17, 19] and the analytical expression for the induced electron number $N_{0}$ was obtained in Ref. [19].

On the other hand, in the supercritical regime $|g|>$ $1 / 2$, the stronger singularity of the Coulomb potential at the origin has to be regularized, for example, by allowing a finite size for charged impurity [7]. However, as long as low-energy physics is concerned, all different regularization can be parametrized by a single quantity $r_{j}^{*}$ per supercritical angular momentum channel $|j|<|g|$ through a boundary condition on the wave function at the origin [25]. As we will find in Eq. (12b), this lowenergy parameter $r_{j}^{*}$ with the dimension of length is de- 
fined only up to multiplicative factors of $e^{\pi / \sqrt{g^{2}-j^{2}}}$. As a consequence, each supercritical channel exhibits the discrete scale invariance and the induced electron density now has a form

$$
n(\boldsymbol{r})=N_{0} \delta(\boldsymbol{r})+\sum_{|j|<|g|} \frac{F_{j}\left(r / r_{j}^{*}\right)}{r^{2}},
$$

where $F_{j}\left(r / r_{j}^{*}\right)$ is an unknown but log-periodic function satisfying $F_{j}\left(r / r_{j}^{*}\right)=F_{j}\left(e^{n \pi / \sqrt{g^{2}-j^{2}}} r / r_{j}^{*}\right)$ with $n$ being an arbitrary integer. Therefore, the vacuum polarization induced by the supercritical Coulomb potential has a power-law tail $\sim 1 / r^{2}$ [15, 16] whose coefficient is a sum of log-periodic functions with respect to the distance from the potential center. Although the coefficient of the power-law tail was considered to be a constant in Refs. [15, 16], our explicit calculation will show that $F_{j}\left(r / r_{j}^{*}\right)$ is the universal log-periodic function presented in Eq. (27).

\section{GREEN'S FUNCTION}

In order to determine the coefficient of the power-law tail in the induced electron density (4), it is more convenient to employ a Green's function method rather than directly dealing with the wave function in Eq. (11) [26, [19]. The single-particle Green's function $G\left(\epsilon ; \boldsymbol{r}, \boldsymbol{r}^{\prime}\right)$ defined for an arbitrary $\epsilon \in \mathbb{C}$ is a solution to

$$
\left(\epsilon+i \boldsymbol{\partial} \cdot \boldsymbol{\sigma}+\frac{g}{r}-m \sigma_{z}\right) G\left(\epsilon ; \boldsymbol{r}, \boldsymbol{r}^{\prime}\right)=\delta\left(\boldsymbol{r}-\boldsymbol{r}^{\prime}\right) \mathbb{1},
$$

where the mass term $m \sigma_{z}$ is introduced to serve as an infrared cutoff and will be set to zero at the end of calculations [3, 19]. By substituting its partial-wave expansion

$$
\begin{aligned}
G\left(\epsilon ; \boldsymbol{r}, \boldsymbol{r}^{\prime}\right)= & \sum_{j=-\infty}^{\infty}\left(\begin{array}{cc}
e^{i(j-1 / 2) \theta} & 0 \\
0 & i e^{i(j+1 / 2) \theta}
\end{array}\right) \frac{\mathcal{G}_{j}\left(\epsilon ; r, r^{\prime}\right)}{2 \pi \sqrt{r r^{\prime}}} \\
& \times\left(\begin{array}{cc}
e^{-i(j-1 / 2) \theta^{\prime}} & 0 \\
0 & -i e^{-i(j+1 / 2) \theta^{\prime}}
\end{array}\right)
\end{aligned}
$$

into Eq. (5) as well as the polar coordinate representation of the $\delta$ function [26], we find the radial Green's function $\mathcal{G}_{j}\left(\epsilon ; r, r^{\prime}\right)$ to satisfy

$$
\left(\begin{array}{cc}
\epsilon+\frac{g}{r}-m & -\partial_{r}-\frac{j}{r} \\
\partial_{r}-\frac{j}{r} & \epsilon+\frac{g}{r}+m
\end{array}\right) \mathcal{G}_{j}\left(\epsilon ; r, r^{\prime}\right)=\delta\left(r-r^{\prime}\right) \mathbb{1},
$$

where $j= \pm 1 / 2, \pm 3 / 2, \ldots$ is the total angular momentum quantum number.

The analytical expression for $\mathcal{G}_{j}\left(\epsilon ; r, r^{\prime}\right)$ can be obtained in a similar way to the corresponding problem in three dimensions [2, [4, 6]. We first set

$$
\begin{aligned}
\mathcal{G}_{j}\left(\epsilon ; r, r^{\prime}\right)= & \theta\left(r-r^{\prime}\right) \psi_{\epsilon j}^{>}(r)\left[\psi_{\epsilon j}^{<}\left(r^{\prime}\right)\right]^{\mathrm{T}} \\
& +\theta\left(r^{\prime}-r\right) \psi_{\epsilon j}^{<}(r)\left[\psi_{\epsilon j}^{>}\left(r^{\prime}\right)\right]^{\mathrm{T}}
\end{aligned}
$$

with $\psi_{\epsilon j}^{>}(r)=\left[\psi_{\epsilon j \uparrow}^{>}(r), \psi_{\epsilon j \downarrow}^{>}(r)\right]^{\mathrm{T}}$ and $\psi_{\epsilon j}^{<}(r)=$ $\left[\psi_{\epsilon j \uparrow}^{<}(r), \psi_{\epsilon j \downarrow}^{<}(r)\right]^{\mathrm{T}}$ being solutions to the radial Dirac equation:

$$
\left(\begin{array}{cc}
\epsilon+\frac{g}{r}-m & -\partial_{r}-\frac{j}{r} \\
\partial_{r}-\frac{j}{r} & \epsilon+\frac{g}{r}+m
\end{array}\right) \psi_{\epsilon j}(r)=0 .
$$

These two solutions have to be normalized as

$$
\psi_{\epsilon j \uparrow}^{>}(r) \psi_{\epsilon j \downarrow}^{<}(r)-\psi_{\epsilon j \downarrow}^{>}(r) \psi_{\epsilon j \uparrow}^{<}(r)=1
$$

to satisfy Eq. (7) and, in addition, have to be chosen so that the radial Green's function $\mathcal{G}_{j}\left(\epsilon ; r, r^{\prime}\right)$ satisfies appropriate boundary conditions at $r \rightarrow \infty$ and $r \rightarrow 0$ with $r^{\prime}$ fixed. It is obvious from its expression (8) that the long-distance limit is controlled by $\psi_{\epsilon j}^{>}(r)$ which has to be bounded at $r \rightarrow \infty$ :

$$
\lim _{r \rightarrow \infty}\left|\psi_{\epsilon j}^{>}(r)\right|<\infty .
$$

On the other hand, the short-distance limit is controlled by $\psi_{\epsilon j}^{<}(r)$ whose boundary condition at $r \rightarrow 0$ requires different treatment for subcritical and supercritical angular momentum channels [15, 16].

In a subcritical angular momentum channel $|j|>|g|$, the radial Dirac equation (9) admits regular and singular solutions $\psi_{\epsilon j}^{<}(r) \rightarrow r^{ \pm \bar{\gamma}}(j \pm \bar{\gamma}, g)^{\mathrm{T}}$ at $r \rightarrow 0$ with the real exponent $\bar{\gamma} \equiv \sqrt{j^{2}-g^{2}}$. Because low-energy physics is dominated by the regular solution, the relevant boundary condition is to impose

$$
\lim _{r \rightarrow 0} \psi_{\epsilon j}^{<}(r) \propto r^{\bar{\gamma}}\left(\begin{array}{c}
j+\bar{\gamma} \\
g
\end{array}\right) .
$$

On the other hand, in a supercritical angular momentum channel $|j|<|g|$, the above two solutions become oscillatory $\psi_{\epsilon j}^{<}(r) \rightarrow r^{ \pm i \gamma}(j \pm i \gamma, g)^{\mathrm{T}}$ at $r \rightarrow 0$ with the imaginary exponent $i \gamma \equiv i \sqrt{g^{2}-j^{2}}$. Because both solutions are now equally important to low-energy physics, the general solution becomes their superposition which is uniquely specified by imposing the boundary condition:

$$
\lim _{r \rightarrow 0} \psi_{\epsilon j}^{<}(r) \propto\left(\frac{r}{r_{j}^{*}}\right)^{i \gamma}\left(\begin{array}{c}
j+i \gamma \\
g
\end{array}\right)-\left(\frac{r}{r_{j}^{*}}\right)^{-i \gamma}\left(\begin{array}{c}
j-i \gamma \\
g
\end{array}\right) .
$$

We thus find that the solution in each supercritical channel is parametrized by a single quantity $r_{j}^{*}>0$ with the dimension of length. An important point, which seems not to be fully appreciated in previous studies, is that the emergent low-energy parameter $r_{j}^{*}$ is defined only up to multiplicative factors of $e^{\pi / \gamma}$, i.e., $e^{n \pi / \gamma} r_{j}^{*}$ with $n$ being an arbitrary integer corresponds to the same physics. As a consequence, each supercritical channel exhibits the discrete scale invariance, which also emerges in nonrelativistic one-body [25], two-body [27], and three-body [28] problems and can be viewed as a manifestation of the quantum scale anomaly and the renormalization group limit cycle [29, 30]. 
It is then straightforward to construct $\psi_{\epsilon j}^{>}(r)$ and $\psi_{\epsilon j}^{<}(r)$ satisfying the above required conditions (10)-(12) from the following two linearly independent solutions to the radial Dirac equation (9) 31]:

$$
\psi_{\epsilon j}^{(1,2)}(r)=\left(\begin{array}{r}
\sqrt{m+\epsilon}\left[u_{\epsilon j}^{(1,2)}(r)+v_{\epsilon j}^{(1,2)}(r)\right] \\
\operatorname{sgn}(m) \sqrt{m-\epsilon}\left[u_{\epsilon j}^{(1,2)}(r)-v_{\epsilon j}^{(1,2)}(r)\right]
\end{array}\right)
$$

with

$$
\begin{aligned}
u_{\epsilon j}^{(1)}(r)= & \frac{g \frac{\epsilon}{\kappa}+\bar{\gamma}}{2 \kappa} \frac{\Gamma\left(1-g \frac{\epsilon}{\kappa}+\bar{\gamma}\right)}{\Gamma(1+2 \bar{\gamma})} \\
& \times(2 \kappa r)^{\bar{\gamma}} e^{-\kappa r} M\left(-g \frac{\epsilon}{\kappa}+\bar{\gamma}, 1+2 \bar{\gamma}, 2 \kappa r\right), \\
v_{\epsilon j}^{(1)}(r)= & \frac{j-g \frac{m}{\kappa}}{2 \kappa} \frac{\Gamma\left(1-g \frac{\epsilon}{\kappa}+\bar{\gamma}\right)}{\Gamma(1+2 \bar{\gamma})} \\
& \times(2 \kappa r)^{\bar{\gamma}} e^{-\kappa r} M\left(1-g \frac{\epsilon}{\kappa}+\bar{\gamma}, 1+2 \bar{\gamma}, 2 \kappa r\right)
\end{aligned}
$$

and

$$
\begin{aligned}
& u_{\epsilon j}^{(2)}(r)=\frac{-1}{j-g \frac{m}{\kappa}}(2 \kappa r)^{\bar{\gamma}} e^{-\kappa r} U\left(-g \frac{\epsilon}{\kappa}+\bar{\gamma}, 1+2 \bar{\gamma}, 2 \kappa r\right), \\
& v_{\epsilon j}^{(2)}(r)=(2 \kappa r)^{\bar{\gamma}} e^{-\kappa r} U\left(1-g \frac{\epsilon}{\kappa}+\bar{\gamma}, 1+2 \bar{\gamma}, 2 \kappa r\right) .
\end{aligned}
$$

Here $\kappa \equiv \sqrt{m^{2}-\epsilon^{2}}$ is introduced, $M(a, b, z)$ and $U(a, b, z)$ are the confluent hypergeometric functions of the first and second kind [32], respectively, and $\bar{\gamma}$ for $|j|>|g|$ should be understood as $\bar{\gamma}=i \gamma$ when $|j|<|g|$. By setting

$$
\psi_{\epsilon j}^{>}(r)=\psi_{\epsilon j}^{(2)}(r)
$$

and

$$
\psi_{\epsilon j}^{<}(r)=\psi_{\epsilon j}^{(1)}(r)+C_{\epsilon j} \psi_{\epsilon j}^{(2)}(r),
$$

the normalization condition (10) and the long-distance boundary condition (11) are satisfied. The so far arbitrary prefactor $C_{\epsilon j}$ is chosen to satisfy the short-distance boundary condition (12), which leads to $C_{\epsilon j}=0$ for a subcritical angular momentum channel $|j|>|g|$ and

$$
C_{\epsilon j}=\frac{-\frac{j-g \frac{m}{\kappa}}{2 \kappa} \frac{j-g \frac{m+\epsilon}{\kappa}-i \gamma}{\left(2 \kappa r_{j}^{*}\right)^{-i \gamma}} \frac{\Gamma\left(1-g \frac{\epsilon}{\kappa}+i \gamma\right)}{\Gamma(2 i \gamma)}}{\frac{j-g \frac{m+\epsilon}{\kappa}+i \gamma}{\left(2 \kappa r_{j}^{*}\right)^{i \gamma}} \frac{\Gamma(1+2 i \gamma)}{\Gamma\left(1-g \frac{\epsilon}{\kappa}+i \gamma\right)}-\frac{j-g \frac{m+\epsilon}{\kappa}-i \gamma}{\left(2 \kappa r_{j}^{*}\right)^{-i \gamma}} \frac{\Gamma(1-2 i \gamma)}{\Gamma\left(1-g \frac{\epsilon}{\kappa}-i \gamma\right)}}
$$

for a supercritical angular momentum channel $|j|<|g|$. Accordingly, the single-particle Green's function (6) is completely determined. For later use, we decompose the radial Green's function into two parts as

$$
\mathcal{G}_{j}\left(\epsilon ; r, r^{\prime}\right)=\mathcal{G}_{j}^{0}\left(\epsilon ; r, r^{\prime}\right)+\delta \mathcal{G}_{j}\left(\epsilon ; r, r^{\prime}\right)
$$

with

$$
\begin{aligned}
\mathcal{G}_{j}^{0}\left(\epsilon ; r, r^{\prime}\right) \equiv & \theta\left(r-r^{\prime}\right) \psi_{\epsilon j}^{(2)}(r)\left[\psi_{\epsilon j}^{(1)}\left(r^{\prime}\right)\right]^{\mathrm{T}} \\
& +\theta\left(r^{\prime}-r\right) \psi_{\epsilon j}^{(1)}(r)\left[\psi_{\epsilon j}^{(2)}\left(r^{\prime}\right)\right]^{\mathrm{T}}
\end{aligned}
$$

and

$$
\delta \mathcal{G}_{j}\left(\epsilon ; r, r^{\prime}\right) \equiv C_{\epsilon j} \psi_{\epsilon j}^{(2)}(r)\left[\psi_{\epsilon j}^{(2)}\left(r^{\prime}\right)\right]^{\mathrm{T}},
$$

where the low-energy parameter $r_{j}^{*}$ with the dimension of length appears only in the latter through $C_{\epsilon j}$.

\section{VACUUM POLARIZATION}

We are now ready to evaluate the vacuum polarization electron density (2), which is expressed in terms of the single-particle Green's function ([6) as

$$
\tilde{n}(\boldsymbol{r})=\sum_{j=-\infty}^{\infty} \int_{-i \infty}^{i \infty} \frac{d \epsilon}{2 \pi i} \frac{\operatorname{Tr}\left[\mathcal{G}_{j}(\epsilon ; \boldsymbol{r}, \boldsymbol{r})\right]}{2 \pi r},
$$

where the contour of the integration over $\epsilon$ is deformed to coincide with the imaginary axis $[2[6,19]$. This formal expression contains divergence which has to be renormalized. In order to separate out the divergent part from the convergent part, we use the decomposition (19) to rewrite the bare electron density (22) as $\tilde{n}(\boldsymbol{r})=\tilde{n}_{0}(\boldsymbol{r})+\sum_{|j|<|g|} \delta \tilde{n}_{j}(\boldsymbol{r})$ with

$$
\tilde{n}_{0}(\boldsymbol{r}) \equiv \sum_{j=-\infty}^{\infty} \int_{-i \infty}^{i \infty} \frac{d \epsilon}{2 \pi i} \frac{\operatorname{Tr}\left[\mathcal{G}_{j}^{0}(\epsilon ; r, r)\right]}{2 \pi r}
$$

and

$$
\delta \tilde{n}_{j}(\boldsymbol{r}) \equiv \int_{-i \infty}^{i \infty} \frac{d \epsilon}{2 \pi i} \frac{\operatorname{Tr}\left[\delta \mathcal{G}_{j}(\epsilon ; r, r)\right]}{2 \pi r},
$$

which have to be treated separately. The first part $\tilde{n}_{0}(\boldsymbol{r})$ is divergent and thus needs the renormalization by requiring the total induced electron number to vanish [2, 3, 5, 19]. Technically, this renormalization can be performed by considering the Fourier transform of the bare electron density $\tilde{\nu}_{0}(\boldsymbol{k})=\int d \boldsymbol{r} e^{-i \boldsymbol{k} \cdot \boldsymbol{r}} \tilde{n}_{0}(\boldsymbol{r})$ with an ultraviolet cutoff $|\epsilon|<\Lambda$ and then introducing the renormalized quantity by $\nu_{0}(\boldsymbol{k})=\lim _{\Lambda \rightarrow \infty}\left[\tilde{\nu}_{0}(\boldsymbol{k})-\lim _{k \rightarrow 0} \tilde{\nu}_{0}(\boldsymbol{k})\right]$ to satisfy the required neutrality condition $\nu_{0}(\mathbf{0})=0$. Because the mass $m$ is the only dimensionful parameter existing in $\mathcal{G}_{j}^{0}(\epsilon ; r, r)$, the resulting dimensionless function $\nu_{0}(\boldsymbol{k})$ can depend only on the ratio $k / m$. Accordingly, it becomes just a constant in the massless limit, $\lim _{m \rightarrow 0} \nu_{0}(\boldsymbol{k})=N_{0}[17,19$, whose inverse Fourier transform gives the cutoff $(m, \Lambda)$ independent renormalized electron density $n_{0}(\boldsymbol{r})=\int d \boldsymbol{k} /(2 \pi)^{2} e^{i \boldsymbol{k} \cdot \boldsymbol{r}} \lim _{m \rightarrow 0} \nu_{0}(\boldsymbol{k})$ as

$$
n_{0}(\boldsymbol{r})=N_{0} \delta(\boldsymbol{r}),
$$

which does not contribute to the power-law tail. 
On the other hand, the second part $\delta \tilde{n}_{j}(\boldsymbol{r})$ is convergent and thus does not need the renormalization because its integrand $\delta \mathcal{G}_{j}(\epsilon ; r, r)$ decreases exponentially at $\kappa r \rightarrow \infty$ [see Eq. (15)]. Accordingly, its contribution to the induced electron density $\delta n_{j}(\boldsymbol{r})=\lim _{m \rightarrow 0} \delta \tilde{n}_{j}(\boldsymbol{r})$ can be directly evaluated by taking the massless limit and is found to have the power-law form

$$
\delta n_{j}(\boldsymbol{r})=\frac{F_{j}\left(r / r_{j}^{*}\right)}{r^{2}}
$$

with the dimensionless coefficient given by

$$
\begin{aligned}
& F_{j}\left(r / r_{j}^{*}\right)=\frac{\gamma}{2 \pi^{2}} \operatorname{Re} \int_{0}^{\infty} d z \frac{\Gamma(1-i g+i \gamma) \Gamma(1-i g-i \gamma)}{\Gamma(1+2 i \gamma) \Gamma(1-2 i \gamma)} \\
& \times\left[\frac{1+\frac{(j-i g+i \gamma) \Gamma(1+2 i \gamma) \Gamma(1-i g-i \gamma)}{(j-i g-i \gamma) \Gamma(1-2 i \gamma) \Gamma(1-i g+i \gamma)}\left(\frac{r}{z r_{j}^{*}}\right)^{2 i \gamma}}{1-\frac{(j-i g+i \gamma) \Gamma(1+2 i \gamma) \Gamma(1-i g-i \gamma)}{(j-i g-i \gamma) \Gamma(1-2 i \gamma) \Gamma(1-i g+i \gamma)}\left(\frac{r}{z r_{j}^{*}}\right)^{2 i \gamma}}\right] \\
& \times e^{-z} U(-i g+i \gamma, 1+2 i \gamma, z) U(1-i g-i \gamma, 1-2 i \gamma, z) .
\end{aligned}
$$

By summing up all the contributions from Eqs. (25) and (26), we obtain the renormalized electron density as

$$
n(\boldsymbol{r})=n_{0}(\boldsymbol{r})+\sum_{|j|<|g|} \delta n_{j}(\boldsymbol{r}),
$$

which establishes the form of the induced electron density presented in Eq. (4). In particular, we find that the coefficient of the power-law tail (27) is log-periodic $F_{j}\left(r / r_{j}^{*}\right)=F_{j}\left(e^{n \pi / \gamma} r / r_{j}^{*}\right)$ as it must be because $e^{n \pi / \gamma} r_{j}^{*}$ with $n$ being an arbitrary integer corresponds to the same physics [see Eq. (12b)] and also universal in the sense that all microscopic details are parametrized by the single quantity $r_{j}^{*}>0$ per supercritical angular momentum channel $|j|<|g|$. Figure 1 shows the obtained universal log-periodic function in the normalized form $2 \pi^{2} F_{j}\left(r / r_{j}^{*}\right) / \gamma$ by taking $j=1 / 2$ and $g=4 / 3$ as an example. Its mean value can be extracted by replacing the whole expression in the square bracket of Eq. (27) with $\operatorname{sgn}(g)$, which leads to

$$
\overline{F_{j}\left(r / r_{j}^{*}\right)}=\frac{\gamma}{2 \pi^{2}} \operatorname{sgn}(g)
$$

in agreement with the constant coefficient of the powerlaw tail considered in Ref. [16]. While $F_{j}\left(r / r_{j}^{*}\right)$ and its mean value (29) coincide in the limit $|g| \rightarrow \infty$, they significantly deviate especially when $|g| \simeq|j|$. We also note that $F_{j}\left(r / r_{j}^{*}\right)$ is odd with respect to $g \rightarrow-g$ and $F_{-j}\left(r / r_{-j}^{*}\right)$ is essentially the same function as $F_{j}\left(r / r_{j}^{*}\right)$ because their apparent difference can be absorbed by redefining $r_{-j}^{*}$.

The phase of the log-periodic oscillation in Eq. (27) is fixed by the nonuniversal parameter $r_{j}^{*}$ which depends on microscopic physics and thus cannot be determined from our perspective of low-energy effective theory. However, it can be related to other physical quantities such as the energy and width of so-called atomic collapse resonances [12]. Although bound states cannot be formed

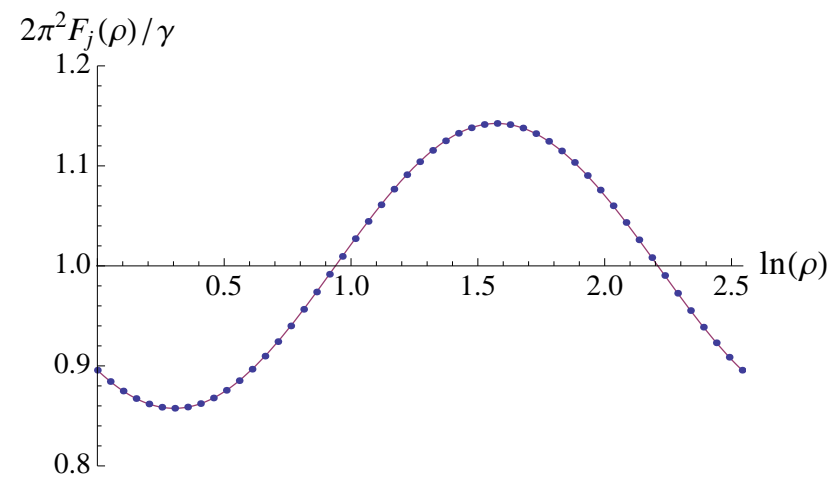

FIG. 1. Universal log-periodic function $2 \pi^{2} F_{j}(\rho) / \gamma$ obtained in Eq. (27) as a function of $\ln (\rho)$. One period in a range $0 \leq$ $\ln (\rho) \leq \pi / \gamma \approx 2.55$ is shown for $j=1 / 2$ and $g=4 / 3$. Dots are the exact result and the solid curve is a fit with a single sine function; $2 \pi^{2} F_{j}(\rho) / \gamma \simeq 1-0.142 \sin [2 \gamma \ln (\rho)+0.821]$.

in the massless limit, it was theoretically shown that an infinite family of resonances emerges in each supercritical angular momentum channel $|j|<|g|[15,33$, 34]. Their energy and width are determined by poles of the singleparticle Green's function (6) in the second Riemann sheet of the complex $\epsilon$ plane, which can arise only as poles of the prefactor $C_{\epsilon j}$ obtained in Eq. (18). By substituting $\kappa \rightarrow-\sqrt{m^{2}-\epsilon^{2}}$ and taking the massless limit $m \rightarrow 0$, we find an infinite family of complex poles at

$$
\epsilon_{j}^{*}=\frac{i}{2 r_{j}^{*}}\left[\frac{(j-i g+i \gamma) \Gamma(1+2 i \gamma) \Gamma(1-i g-i \gamma)}{(j-i g-i \gamma) \Gamma(1-2 i \gamma) \Gamma(1-i g+i \gamma)}\right]^{\frac{1}{2 i \gamma}}
$$

with multiplicative factors of $e^{\pi / \gamma}$, which leads to

$$
E_{j}^{(n)}-\frac{i}{2} \Gamma_{j}^{(n)}=\hbar v_{\mathrm{F}} e^{-n \pi / \gamma} \epsilon_{j}^{*}
$$

as the energy and width of the $n$th atomic collapse resonance. Therefore, if $r_{j}^{*}$ for a given system is determined through the energy or width of an atomic collapse resonance (31), we then have an unambiguous prediction for the power-law tail of the vacuum polarization electron density (27), and vice versa. Furthermore, the complex expression in the square bracket of Eq. (27) can be greatly simplified by using the complex energy in Eq. (31) as

$$
[\cdots]=\left[\frac{1+\left(\frac{2 \epsilon_{j}^{*} r}{i z}\right)^{2 i \gamma}}{1-\left(\frac{2 \epsilon_{j}^{*} r}{i z}\right)^{2 i \gamma}}\right]
$$

which model-independently relates the two intriguing phenomena caused by the supercritical Coulomb potential, i.e., the vacuum polarization and the atomic collapse resonances.

Finally, it is worthwhile to compare our prediction with the induced electron density computed for the tightbinding Hamiltonian on a honeycomb lattice whose lowenergy physics is described by two valley species of massless Dirac fermions [35, 36]. Figure 2 shows numerical 


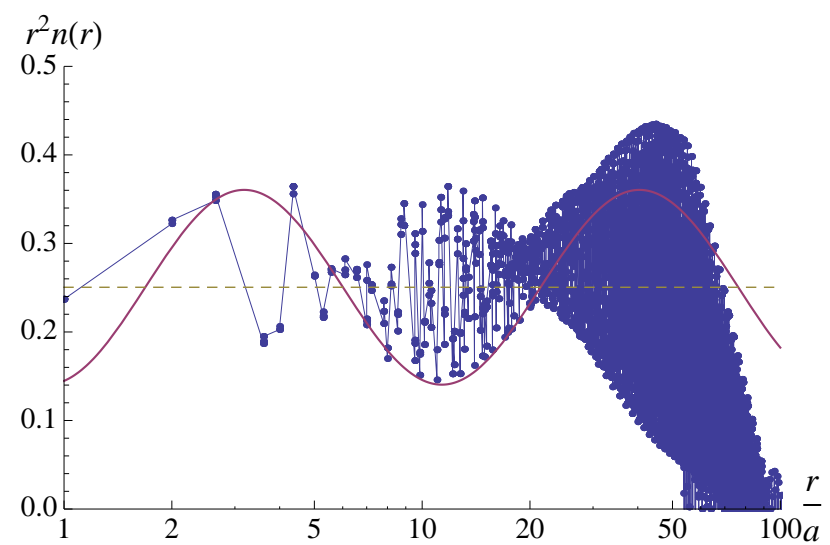

FIG. 2. Induced electron density $n(\boldsymbol{r})$ for the tight-binding Hamiltonian on a honeycomb lattice as a function of $r$ in units of the lattice parameter $a$. Connected dots are numerical data for $g=4 / 3$ obtained in Ref. [15] by the exact diagonalization with $124^{2}$ lattice sites. The solid curve is a fit based on our prediction (33) with the mean value $2 \gamma / \pi^{2} \approx 0.25$ indicated by the horizontal dashed line.

data for $g=4 / 3$ obtained in Ref. [15] by the exact diagonalization with $124^{2}$ lattice sites in units of the lattice parameter $a$. In addition to rapid oscillations presumably caused by the lattice cutoff, there seems to be a slow oscillation which should be contrasted with the predicted log-periodic oscillation. Because only $j= \pm 1 / 2$ channels are supercritical for $g=4 / 3$, our prediction (4) reduces to $r^{2} n(\boldsymbol{r})=2 \sum_{j= \pm 1 / 2} F_{j}\left(r / r_{j}^{*}\right)$ including the factor two due to the valley degeneracy. Here the universal log-periodic function $F_{j}\left(r / r_{j}^{*}\right)$, as seen in Fig. 1. can be excellently approximated by a single sine function with a relative error less than $0.01 \%$. Accordingly, our prediction for the power-law tail of the induced electron density is expressed as

$$
r^{2} n(\boldsymbol{r}) \simeq \frac{2 \gamma}{\pi^{2}}-A \sin \left[2 \gamma \ln \left(\frac{r}{a}\right)+\varphi\right],
$$

where the amplitude $A$ and the phase $\varphi$ are related to the unknown two low-energy parameters $r_{ \pm 1 / 2}^{*} / a$. We find in Fig. 2 that the lower envelope of numerical data in the intermediate region $a \ll r \ll 50 a$ between the lattice cutoff and the system radius is well fitted by choosing $A \approx 0.11$ and $\varphi \approx 1.85$, which supports the validity of our prediction.

\section{CONCLUDING REMARKS}

In this paper, we studied massless Dirac fermions in a supercritical Coulomb potential with the emphasis on that its low-energy physics is universal and each supercritical angular momentum channel exhibits the discrete scale invariance. In particular, we showed that the induced vacuum polarization has a power-law tail whose coefficient is a sum of log-periodic functions with respect to the distance from the potential center. This coefficient can also be expressed in terms of the energy and width of so-called atomic collapse resonances. While these universal features are explicitly demonstrated only in two dimensions [see Eqs. (26)-(32)], it is straightforward to extend our present analysis to three dimensions as well. Therefore, qualitatively the same features are indeed expected in the vacuum polarization caused by a superheavy atomic nucleus with $Z>\alpha^{-1}$ as long as ultraviolet and infrared cutoffs (i.e., nuclear charge radius and electron Compton wavelength, respectively) are well separated compared to log-periodic oscillations, which shed new light on the longstanding fundamental problem of quantum electrodynamics. Furthermore, because lowenergy physics of graphene is described by massless Dirac fermions in two dimensions, our universal predictions on the vacuum polarization and its relationship to observed atomic collapse resonances [12] can in principle be tested experimentally by measuring the induced electron density with scanning probe microscopy techniques [37].

While the electron-electron interaction has been neglected in our present analysis, our novel finding on the vacuum polarization may be useful to develop further insight into the screening of the supercritical Coulomb impurity in the presence of the electron-electron interaction. One possible approach is to write down the selfconsistent renormalization group equation in the same spirit as Ref. [16]:

$$
\frac{d Z_{\mathrm{eff}}(R)}{d \ln R}=-2 \pi\left[\sum_{|j|<|g|} F_{j}\left(R / r_{j}^{*}\right)\right]_{Z \rightarrow Z_{\mathrm{eff}}(R)},
$$

where $Z_{\text {eff }}(R) \equiv Z-\int_{|\boldsymbol{r}|<R} d \boldsymbol{r} n(\boldsymbol{r})$ multiplied by $e$ is the total charge within the radius $R$. Because the right hand side of Eq. (34) is negative, the total charge decreases as the radius increases until the right hand side vanishes, i.e., $\left|g_{\text {eff }}(R)\right| \rightarrow 1 / 2$, which leads to the screening of the supercritical charge down to the critical value [16]. Detailed analysis of the solution to our self-consistent renormalization group equation (34) shall be deferred to a future work.

\section{ACKNOWLEDGMENTS}

The author thanks Vitor M. Pereira for providing numerical data in Ref. [15] and related discussions. This work was supported by JSPS KAKENHI Grant Number 25887020 . 
[1] Ya. B. Zeldovich and V. S. Popov, Usp. Fiz. Nauk 105, 403 (1971) [Sov. Phys. Usp. 14, 673 (1972)].

[2] E. Wichmann and N. M. Kroll, Phys. Rev. 96, 232 (1954); Phys. Rev. 101, 843 (1956).

[3] L. S. Brown, R. N. Cahn, and L. D. McLerran, Phys. Rev. Lett. 32, 562 (1974); Phys. Rev. Lett. 33, 1591 (1974); Phys. Rev. D 12, 581 (1975); Phys. Rev. D 12, 596 (1975); Phys. Rev. D 12, 609 (1975).

[4] M. Gyulassy, Phys. Rev. Lett. 32, 1393 (1974); Phys. Rev. Lett. 33, 921 (1974); Nucl. Phys. A 244, 497 (1975).

[5] A. I. Mil'shtein and V. M. Strakhovenko, Phys. Lett. A 90, 447 (1982); Phys. Lett. A 92, 381 (1982); Zh. Eksp. Teor. Fiz. 84, 1247 (1983) [Sov. Phys. JETP 57, 722 (1983)].

[6] W. Greiner, B. Müller, and J. Rafelski, Quantum Electrodynamics of Strong Fields (Springer-Verlag, Berlin, 1985).

[7] I. Ya. Pomeranchuk and Y. A. Smorodinsky, J. Phys. USSR 9, 97 (1945).

[8] K. S. Novoselov, A. K. Geim, S. V. Morozov, D. Jiang, Y. Zhang, S. V. Dubonos, I. V. Grigorieva, and A. A. Firsov, Science 306, 666 (2004).

[9] K. S. Novoselov, D. Jiang, F. Schedin, T. J. Booth, V. V. Khotkevich, S. V. Morozov, and A. K. Geim, Proc. Natl. Acad. Sci. USA 102, 10451 (2005).

[10] A. H. Castro Neto, F. Guinea, N. M. R. Peres, K. S. Novoselov, and A. K. Geim, Rev. Mod. Phys. 81, 109 (2009).

[11] Y. Wang, V. W. Brar, A. V. Shytov, Q. Wu, W. Regan, H.-Z. Tsai, A. Zettl, L. S. Levitov, and M. F. Crommie, Nat. Phys. 8, 653 (2012).

[12] Y. Wang, D. Wong, A. V. Shytov, V. W. Brar, S. Choi, Q. Wu, H.-Z. Tsai, W. Regan, A. Zettl, R. K. Kawakami, S. G. Louie, L. S. Levitov, and M. F. Crommie, Science 340, 734 (2013).

[13] A. Luican-Mayer, M. Kharitonov, G. Li, C.-P. Lu, I. Skachko, A.-M. B. Gonçalves, K. Watanabe, T. Taniguchi, and E. Y. Andrei, Phys. Rev. Lett. 112, 036804 (2014).

[14] M. I. Katsnelson, Phys. Rev. B 74, 201401(R) (2006).

[15] V. M. Pereira, J. Nilsson, and A. H. Castro Neto, Phys. Rev. Lett. 99, 166802 (2007).
[16] A. V. Shytov, M. I. Katsnelson, and L. S. Levitov, Phys. Rev. Lett. 99, 236801 (2007).

[17] R. R. Biswas, S. Sachdev, and D. T. Son, Phys. Rev. B 76, 205122 (2007).

[18] M. M. Fogler, D. S. Novikov, and B. I. Shklovskii, Phys. Rev. B 76, 233402 (2007).

[19] I. S. Terekhov, A. I. Milstein, V. N. Kotov, and O. P. Sushkov, Phys. Rev. Lett. 100, 076803 (2008).

[20] V. N. Kotov, B. Uchoa, and A. H. Castro Neto, Phys. Rev. B 78, 035119 (2008).

[21] V. N. Kotov, V. M. Pereira, and B. Uchoa, Phys. Rev. B 78, 075433 (2008).

[22] V. M. Pereira, V. N. Kotov, and A. H. Castro Neto, Phys. Rev. B 78, 085101 (2008).

[23] For a comprehensive review, see Sec. IV in V. N. Kotov, B. Uchoa, V. M. Pereira, F. Guinea, and A. H. Castro Neto, Rev. Mod. Phys. 84, 1067 (2012).

[24] P. A. Maksym and H. Aoki, J. Phys.: Conf. Ser. 456, 012026 (2013).

[25] K. M. Case, Phys. Rev. 80, 797 (1950).

[26] See, for example, Delta Function in Wolfram MathWorld: http://mathworld.wolfram.com/DeltaFunction.html.

[27] Y. Nishida and D. Lee, Phys. Rev. A 86, 032706 (2012).

[28] V. Efimov, Phys. Lett. B 33, 563 (1970).

[29] A. Gorsky and F. Popov, Phys. Rev. D 89, 061702(R) (2014).

[30] K. M. Bulycheva and A. S. Gorsky, Usp. Fiz. Nauk 184, $182(2014)$

[31] D. S. Novikov, Phys. Rev. B 76, 245435 (2007).

[32] See, for example, Handbook of Mathematical Functions, edited by M. Abramowitz and I. A. Stegun (Dover, New York, 1965), Chap. 13.

[33] A. V. Shytov, M. I. Katsnelson, and L. S. Levitov, Phys. Rev. Lett. 99, 246802 (2007).

[34] A. Shytov, M. Rudner, N. Gu, M. Katsnelson, and L. Levitov, Solid State Commun. 149, 1087 (2009).

[35] G. W. Semenoff, Phys. Rev. Lett. 53, 2449 (1984).

[36] D. P. DiVincenzo and E. J. Mele, Phys. Rev. B 29, 1685 (1984).

[37] A. Deshpande and B. J. LeRoy, Physica E 44, 743 (2012). 